\title{
Hereditary Breast Cancer Genetics-From Clinical Curiosities to Mainstream Paradigms
}

\author{
G. J. Lindeman • J. E. Visvader
}

Published online: 25 March 2011

(C) Springer Science+Business Media, LLC 2011

One of the first descriptions of hereditary breast cancer dates back to an insightful publication in 1757 by an eminent French surgeon, Le Dran [1]. Here he described breast cancer arising in a 19 year-old nun from Avignon, whose grandmother and a maternal great uncle had both succumbed from the disease. The patient initially refused mastectomy, apparently convinced that "her blood was corrupted by a cancerous ferment natural to her family" [2]. In 1866, another French surgeon, Broca, described four generations of breast cancer in his wife's family pedigree [3]. Moving forward to the next century, Lynch and colleagues described 34 families with hereditary breast/ ovarian carcinoma syndrome [4].

These clinical curiosities might at first seem far removed from mainstream oncology. However, over the last 20 years a profound increase in knowledge of the molecular and cellular underpinnings of hereditary breast cancer has occurred. Precisely when this 'modern era' began is difficult to define, but landmarks include the discovery of germline mutations in the tumor suppressor p53 in kindreds with Li-Fraumeni syndrome [5], where early-onset breast cancer is a hallmark feature. At about the same time, MaryClaire King and colleagues localized the BRCA1 locus to

\author{
G. J. Lindeman $(\bowtie) \cdot J$. E. Visvader \\ Stem Cells and Cancer Division, The Walter and Eliza Hall \\ Institute of Medical Research, \\ $1 \mathrm{G}$ Royal Parade, \\ Parkville, VIC 3052, Australia \\ e-mail: lindeman@wehi.edu.au \\ J. E. Visvader \\ e-mail: visvader@wehi.edu.au \\ G. J. Lindeman \\ Familial Cancer Centre, The Royal Melbourne Hospital, \\ Grattan Street, \\ Parkville, VIC 3050, Australia
}

$17 \mathrm{q} 21$ [6], thereby triggering a tense race to clone the $B R C A 1$ and BRCA2 genes [7, 8]. Their discovery, in 1994 and 1995 , immediately captivated the attention of scientists, clinicians and the public.

It is interesting to reflect on the rapid reciprocal transfer of research findings between bench and bedside that has ensued, despite the relative rarity of hereditary cancer syndromes. This has been facilitated by a multi-faceted approach taken by researchers with diverse interests encompassing molecular and cell biology, mouse biology, epidemiology and clinical care. In addition, patient advocacy and combined research efforts by international consortia have played seminal roles.

Many of the lessons learnt from rare familial cancer syndromes will almost certainly have relevance in sporadic breast cancer, through somatic silencing of shared signaling pathways, or perturbations in similar target breast epithelial cells. Further research will almost certainly lead to the identification of additional breast cancer-predisposing genes, including better appreciation of low- to moderaterisk modifier genes that play an important role in sporadic breast cancer. Mouse mammary models including xenograft models will continue to play a central role in these research efforts.

This issue of the Journal of Mammary Gland Biology and Neoplasia contains reviews that provide a timely snapshot of some of these issues. The review by Shuen and Foulkes provides an overview of the genetics of breast cancer susceptibility. They note that insights into the role of BRCAI and $B R C A 2$ in maintaining genomic integrity and regulating DNA repair are leading to the development of targeted therapies for patients. The potential importance of risk modifier genes for carriers of $B R C A 1$ and $B R C A 2$ mutations, recently identified through genome-wide association studies, is also discussed. Mutations in BRCA1 and BRCA2 account 
for about $20 \%$ of familial breast cancer, with other high penetrance genes likely accounting for a small additional percentage of cases. The cumulative effects of multiple lowto moderate-risk genes are presumed to account for the remaining cases. Shuen and Foulkes discuss this growing list of genes and their relevance for genetic testing.

An accompanying overview by Clark and Domchek addresses clinical management issues in hereditary cancer syndromes. It is apparent from both reviews that the clinical landscape continues to change quite rapidly, in part due to emerging insights into tumor biology and behavior that are informing optimal management strategies. Moreover, the discovery of distinct genetic lesions is leading to the development of novel targeted therapies, such as PARP inhibitors, now in early phase clinical trials.

Tumor pathology and molecular markers play a central role in recognizing and managing hereditary breast cancer. As outlined in the review by Vargas et al, there is a growing appreciation of genotype-phenotype correlations for certain hereditary cancers. The characteristic 'basal-like' phenotype of BRCA1-associated tumors is a prime example of the power of the pathology to alert clinicians to the potential presence of an underlying germline mutation. Other important genotype-phenotype associations are discussed, including germline $\mathrm{CDH1}$ mutations in invasive lobular cancer and germline $p 53$ mutations associated with very early onset breast tumors carrying HER 2 amplification.

The utility of mouse models to study BRCA1-deficient breast cancer is reviewed by Michalak and Jonkers. Here the authors summarize various mouse models - ranging from xenografts to genetically engineered mice - that have been generated to better understand BRCAl-associated breast cancer. The emerging importance of other genes (such as $P T E N$ or the polycomb-repressive complex gene EZH2) will likely provide additional insights as newer mouse models are developed. The authors outline the importance of mouse models for 'proof-of-principle' pre-clinical studies of novel agents such as the PARP inhibitors. These are also useful for determining mechanisms of acquired drug-resistance in vivo and to study cancer stem cells.

The review by Molyneux and Smalley explores the cellular mechanism(s) behind the genotype-phenotype correlation in BRCAl-asssociated breast cancer. They summarize recent studies in both mice and humans that have identified luminal progenitor cells as 'cells-of-origin' in BRCA1-associated breast cancer. Their review under- scores the potential pitfalls in simply relying on gene profiling studies to distinguish the cell of tumor origin. They demonstrate that Blg-Cre Brcal floxfflox $p 53^{+/-}$mice develop mammary tumors which are remarkably similar to their human BRCAl counterpart, and further illustrate the relevance of mouse models to studying human disease.

Rapid technological advances have enabled genomewide association studies (GWAS) to identify functional sequence variants that modify breast cancer risk. The review by Blackburn and Jerry emphasizes the importance of understanding precisely how SNPs identified by GWAS affect tumor susceptibility. Rodent mammary tumor models provide important tools for understanding the relevance of functional polymorphisms in modulating gene versus environment interactions. They describe several models, including the $\operatorname{Trp} 53^{+/-}$mouse model of Li-Fraumeni Syndrome, where susceptibility to different tumor types is profoundly dependent on genetic background. The DMBA mammary tumor model represents another useful model, whereby sensitivity to hormones, radiation and other environmental factors can be explored. Future challenges will include determining how to best translate findings on low-penetrance risk modifiers (in the presence or absence of high penetrance alleles) to improve breast cancer outcomes.

\section{References}

1. Le Dran H. Mémoire avec un précis de plusieurs observations sur le cancer. Mém Acad R Chir. 1757;3:1-54.

2. Eisinger F, Sobol H, Serin D, Whorton JC. Hereditary breast cancer, circa 1750. Lancet. 1998;351(9112):1366.

3. Broca P. Etiologie des productions accidentelles. In: Asselin P, editor. Traité des tumeurs. Chapter IV. Paris; 1866. p. 142-164.

4. Lynch HT, Krush AJ, Lemon HM, et al. Tumor variation in families with breast cancer. JAMA. 1972;222(13):1631-5.

5. Malkin D, Li FP, Strong LC, et al. Germ line p53 mutations in a familial syndrome of breast cancer, sarcomas, and other neoplasms. Science. 1990;250(4985):1233-8.

6. Hall JM, Lee MK, Newman B, et al. Linkage of early-onset familial breast cancer to chromosome 17q21. Science. 1990;250 (4988):1684-9.

7. Miki Y, Swensen J, Shattuck-Eidens D, et al. A strong candidate for the breast and ovarian cancer susceptibility gene BRCA1. Science. 1994;266(5182):66-71.

8. Wooster R, Bignell G, Lancaster J, et al. Identification of the breast cancer susceptibility gene BRCA2. Nature. 1995;378(6559):78992. 